\title{
USING AUTOMATIC SPEECH RECOGNITION FOR MANIPULATION AND CONTROL OF ROBOTIC PLATFORMS
}

\section{MIGUEL PÉREZ PEREIRA, HOLMAN MONTIEL ARIZA \& HARVEY GÓMEZ CASTILLO}

Universidad Distrital Francisco José de Caldas, Facultad Tecnológica, Bogotá, Colombia

\begin{abstract}
The linking of humanoid robotic platforms in people's daily life is advancing by leaps and bounds, day after day the development and implementation of these technological tools in people's daily activities is more frequent; this fact establishes that the interaction and/or communication process between human-robot must be optimal, and that is where a wide field of research and experimentation appears, allowing a clear and fluid communication between the robot and its respective users. Therefore, this article proposes a basic strategy associated with automatic voice recognition (action commands) for the interaction and control of the humanoid robotic platform NAO. This work describes a solution composed by two operational blocks, the first block refers to the use of the Sphinx CMU Library for the recognition of the action commands for the robot and the second block describes the commands needed for the locomotion process of the platform humanoid NAO.

KEYWORDS: Automatic voice recognition, Manipulation, Humanoid platforms, Collaborative robots Robot programming
\end{abstract}

Received: Oct 03, 2020; Accepted: Oct 23, 2020; Published: Oct 29, 2020; Paper Id.: IJMPERDOCT20203

\section{INTRODUCTION}

One of the fundamental parameters that must be taken into account when using humanoid robotic platforms in care work is communication (Sauppé \& Mutlu, 2014b); although it is true that day by day these technological tools are being incorporated into multiple activities of our daily life, supporting from education tasks, household tasks, to complex tasks in the diverse productive sectors of our society (Kitakoshi, et al., 2017), there is still an arduous path ahead in the face of the multiple and diverse challenges in terms of achieving natural and effective communication between these robots and their respective users (Sauppé \& Mutlu, 2014c).

A vital starting point that take both designers and researchers in the field, are the models of behavior based on studies of human interactions (Sauppé \& Mutlu, 2014a) (Huang \& Mutlu, 2014) (Kennedy, et al., 2017); this in order to achieve the identification of patterns that manage to represent the various rules that can control the behavior of a robot (Montiel, H. et al, 2017). This leads to that to achieve appropriate processes of human-robot interaction generally makes use of the two natural communication channels that we have, the visual and verbal (Burget, et al., 2017).

With respect to the application of visual tools to control or communicate with a robotic platform, a wide variety of works can be found associated with visual pattern recognition, landmarks, motion imitation, hand-eye coordination applications, among many other activities that provide operational support to the functional process of interaction based on these communication channels (Andrist, S. et al, 2014). To identify the implementations associated with the second natural communication channel, the verbal, we can find applications focused on voice 
command detections that can control these robotic platforms (UPase, 2016) (Kozhirbayev, et al, 2018).

The recognition of voice commands associated with the interaction of humanoid robotic platforms such as the NAO must be adapted to the various criteria of emission by the people with whom it interacts, factors such as vowel characters, nasality, tone, articulation, duration; all these possible factors make the problem of Automatic Speech Recognition (ASR), a complex subject of study in the area of digital signal processing linked to the interaction and control of robotic platforms (Rosen, E. et al., 2019).

Taking into account the above, the main objective of this work is to present a first approach to the implementation and use of one of the most used libraries for automatic voice recognition in conjunction with a humanoid robotic platform (NAO); this in order to be evaluated its possible use in assistance robotics tasks and applications that allow improving human-robot interaction, in order to provide new robotic tools to support social, educational and productive environments.

\section{METHODOLOGY}

The proposed development is composed of two main operating blocks: The automatic voice recognition and the robot locomotion block. The development is based on the implementation of the Hidden Models of Markov for the automatic recognition of commands of action that the robot will receive; once it has been identified the instruction (variable String) it is proceeded to the comparison and identification of this order, for later to execute an action of movement already preestablished as command of detected action.

\section{Automatic Speech Recognition (ASR)}

Currently there are three ways to address the problem of ASR, the first is the detection of isolated words, and is that the system focuses on the recognition of isolated words or phrases taken from a database previously determined, these problems are usually the most basic and easy to address by ASR systems. The second problem is the recognition of connected words, and consists of the speaker speaking fluently a succession of words belonging to a restricted vocabulary, although this system requires a greater degree of work than the first problem, the ASR system must solve it by searching a finite database of words, which facilitates recognition, the third problem is that of continuous speech, and consists of the speaker speaking fluently using words from a large vocabulary (usually unlimited), and the ASR system must recognize the words taking into account the fundamental elements of the language and language implemented; Such systems are the most complex ones since they must take into account the language model, the acoustic model, the phoneme recognition, etc., in order to achieve an optimal detection (Nasereddin \& Omari, 2017) (Asemi, A. et al., 2019).

This third problem (continuous speech), is the one that is addressed in this research, since the system proposed here recognizes the continuous speech independent of the speaker integrating acoustic and linguistic knowledge for the movement of the robot, and this is where the technique of the Hidden Markov Model (HMM) stands out as a fundamental tool in the solution of this type of problem.

\section{Hidden Markov Model (HMM)}

Language is an inherently temporary phenomenon. When we understand and produce spoken language, we are processing continuous input flows of undefined length (Keselj, 2009), this characteristic of spoken language is what has allowed positioning HMM as one of the preferred techniques to address this problem. Before talking about the Hidden Markov Models, we should talk about Markov chains. A Markov chain is a model that tells us something about the probabilities of 
succession of random variables, states, each of which can take values from some set, in other words, it is a random process that starts with some defined states, such states can be given by the user and have direct relevance to the problem, the property that a process $\left(\mathrm{X}_{\mathrm{t}}\right)$ should have to be a Markov chain is (Amit, 2019):

$$
\mathbb{P}\left(X_{t}=j \mid X_{1}=i_{1}, \ldots, X_{t-1}=i_{t-1}\right)=\mathbb{P}\left(X_{t}=j \mid X_{t-1}=i_{t-1}\right)
$$

Equation (1) shows that the probability of being in a state " $\mathrm{j}$ " depends only on the previous state " $\mathrm{t}-1$ " and not on the states prior to it, in terms of the problem that concerns us, it means that the probability of the occurrence of the current word depends only on the word prior to it, an undeniable fact in spoken language.

A Markov chain is useful when we need to calculate a probability of occurrence for a sequence of observable events. However, in hidden Markov models (HMM) we have a hidden Markov chain (which we cannot observe), and each state randomly generates one of the observations, which are visible to us (Castro, 2011).

A Hidden Markov Model (HMM) can be represented as a finite state machine, where the state sequences are hidden, and the transitions are given by a state probability function. A hidden Markov model can be presented in various topologies, Figure 1 shows the left-right model or Bakis model ideal for modeling speech recognition.

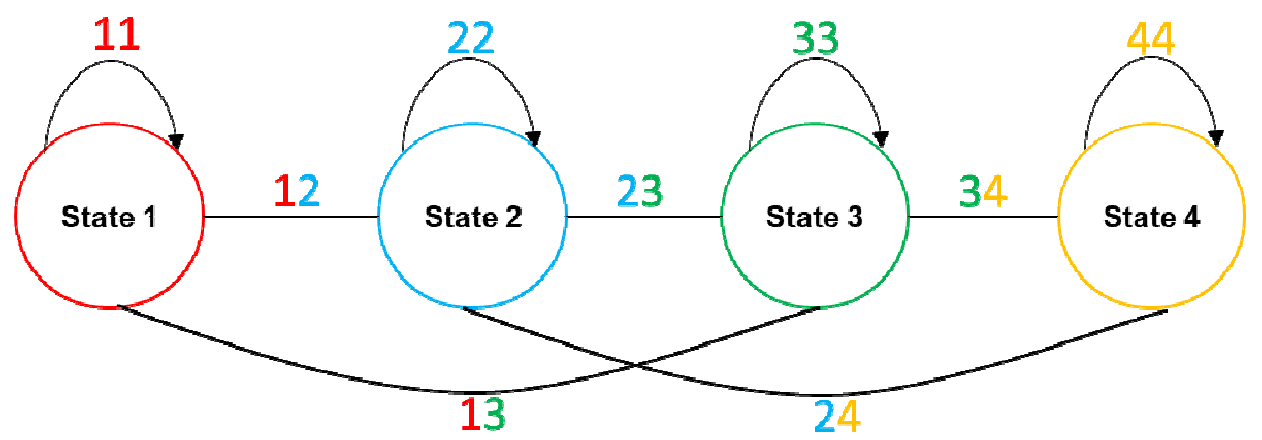

Figure 1: Bakis Model with 4 states

\section{IMPLEMENTATION}

The figure 2 shows the block diagram of the implemented solution, it consists of 4 modules:

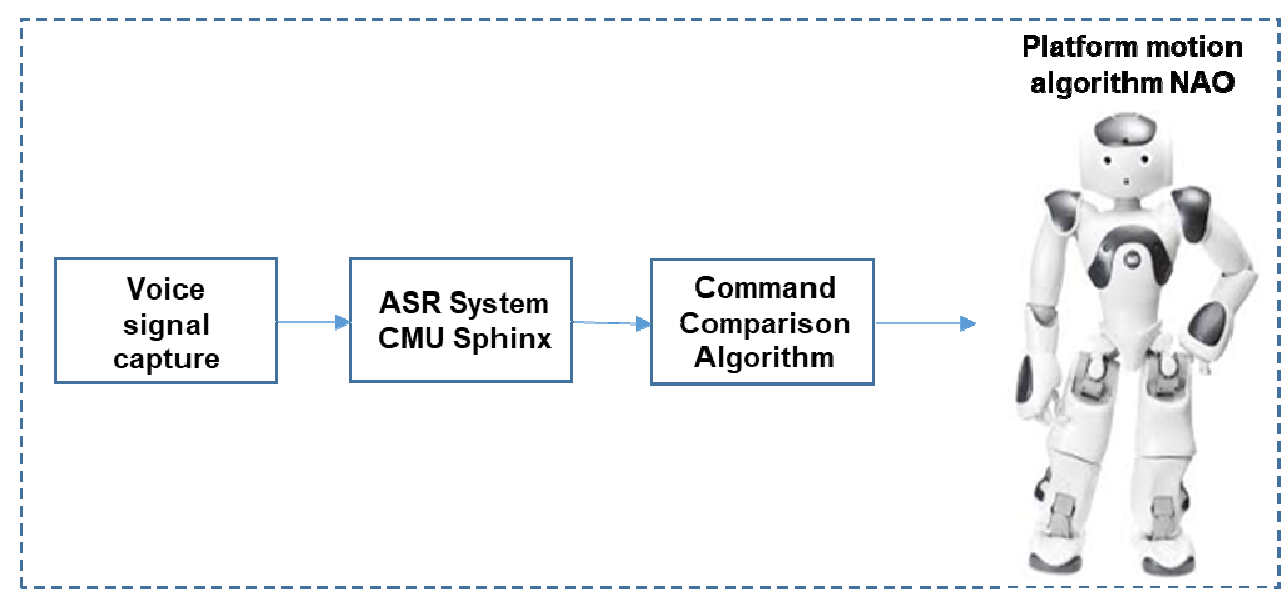

Figure 2: Block Diagram of the Implemented Solution 


\section{Voice Signal Capture}

To capture the voice signal, the microphones brought by the NAO6 platform were implemented. The NAO platform has 4 microphones with sensitivity -12 dBV/PA to $1 \mathrm{KHZ}$ and a frequency range of $100 \mathrm{HZ}$ to $10 \mathrm{KHZ}$ which makes them ideal for capturing voice, these microphones are arranged in the head of the robot so that they work together to obtain an omnidirectional capture pattern, the location of the microphones can be seen in figure 3 .

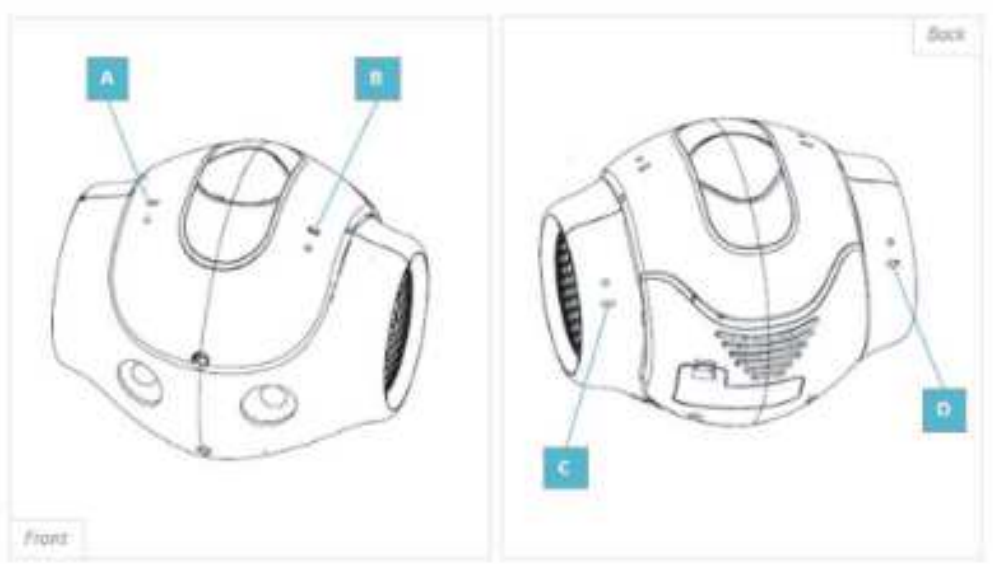

Figure 3: Location of the microphones in the head of the NAO Robot (Softbankrobotics, 2020a)

\section{Sphinx CMU Library for ASR}

CMU Sphinx is a speech recognition system applied to continuous speech, developed at Carnegie Mellon University; this system is based on HMM for recognition, therefore it makes use of acoustic models and language models for this purpose, a general block diagram of the system is shown in figure 4 .

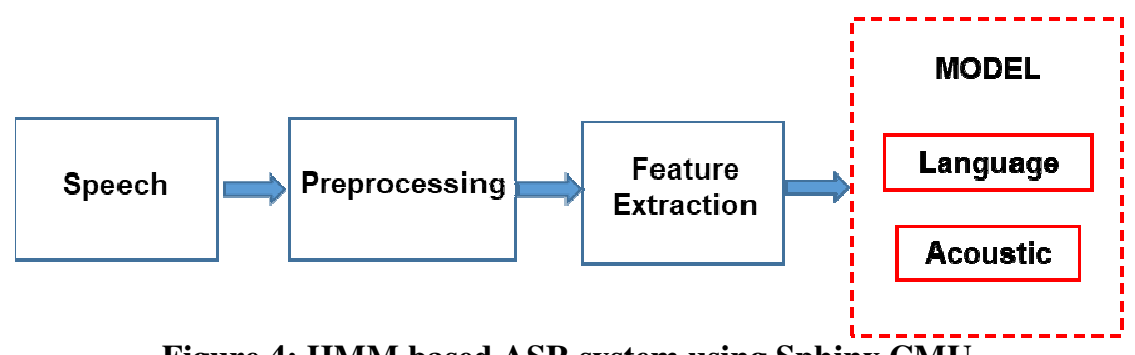

Figure 4: HMM based ASR system using Sphinx CMU

An acoustic model is a central component of any ASR system and establishes a relationship between a sound file and a string representing a phoneme for the language to be detected. On the other hand, a language model defines the structure of the language, in other words, it contains the probability of certain words appearing in a certain order, hence its importance for the HMM algorithm.

The Sphinx CMU Library has the following modules (Maldonado, D. et al, 2016):

- $\quad$ Pocketsphinx: Library lightweight for voice recognition written in C language.

- $\quad$ Sphinxbase: Library base required by the other applications.

- Sphinx4: Adjustable and modifiable recognizer written in Java.

- Sphinxtrain: Tool for training the acoustic model. 
In this research we made use of the Sphinx CMU library with its Sphinxbase and Pocketsphinx modules, as Pocketsphinx is in $\mathrm{C}$ language we installed the interface that will allow us to use pocketsphinx with python called setuptools and wheel. As the Sphinx CMU models and dictionaries are originally for the English language, we downloaded and replaced the corresponding ones for the Spanish language cmusphinx-es-5.2.tar.gz, es-20k.lm.gz, es.dict.

\section{NAO Platform Locomotion}

The NAO humanoid platform has a motion API called ALMotion, which provides a series of easy-to-implement methods to control the stiffness and angles of all its joints in a simple manner, thus allowing full control over the walk of this humanoid robot.

Some of the functions and basic methods more used in Python linked to the locomotion of the robot and considering the documentation delivered by the manufacturer are (Softbankrobotics, 2020b):

void ALMotionProxy::move(const float\& $x$, const float\& $y$, const float\& theta, const AL::ALValue moveConfig)

This method makes the robot move at the given normalized speed, its operating parameters are $x$ - velocity along $\mathrm{X}$-axis, in meters per second. Use negative values for backward motion; $y$ - velocity along Y-axis, in meters per second. Use positive values to go to the left and theta - velocity around Z-axis, in radians per second. Use negative values to turn clockwise.

This displacement method has a possible configuration variant:

- ALMotionProxy::move with move configuration

Where the additional parameter would be moveConfig - An ALValue with the custom move configuration.

bool ALMotionProxy: moveTo(const float\& $x$, const float\& $\mathbf{y}$, const float\& theta)

This function makes the robot move to the pose given in the floor plane, where $x$ - Distance along the $\mathrm{X}$ axis in meters, $y$ - Distance along the $\mathrm{Y}$ axis in meters and theta - Rotation around the $\mathrm{Z}$ axis in radians [-3.1415 to 3.1415]. This displacement function has 3 possible configurations, these are

- ALMotionProxy::moveTo with move configuration

- ALMotionProxy::moveTo with controlPoints

- ALMotionProxy::moveTo with controlPoints and move configuration

For these possible variants the additional parameters would be, moveConfig - An ALValue with the custom move configuration and controlPoint - An ALValue with all the control points [[x1,y1,theta1], ..., [xN,yN,thetaN].

void ALMotionProxy::moveToward(const float\& x, const float\& y, const float\& theta)

This method makes the robot move at the given normalized speed, its operating parameters are $x$ - normalized, unitless, velocity along $\mathrm{X}$-axis. +1 and -1 correspond to the maximum velocity in the forward and backward directions, respectively; $y$ - normalized, unitless, velocity along Y-axis. +1 and -1 correspond to the maximum velocity in the left and right directions, respectively; theta - normalized, unitless, velocity around Z-axis. +1 and -1 correspond to the maximum velocity in the counterclockwise and clockwise directions, respectively. 
Figure 5 shows an example of some motion statements tested on the implementation developed, each statement or control command was validated by an IF statement associated with multiple comparison options.

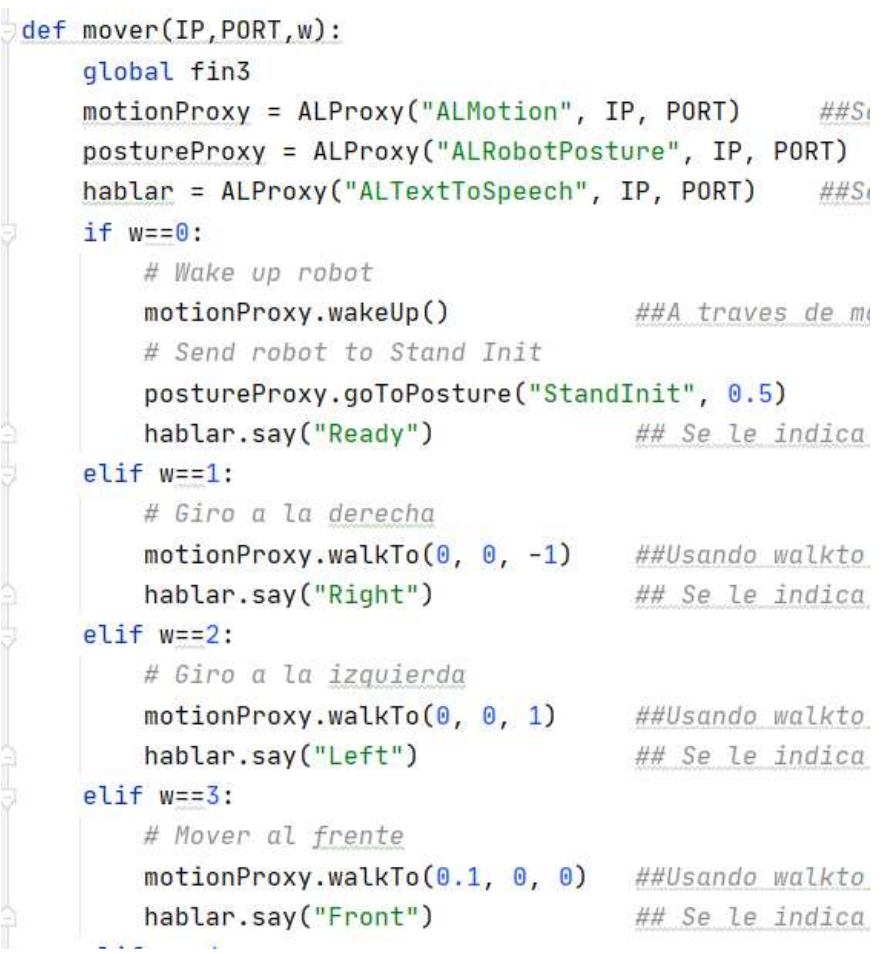

Figure 5: Test code Associated with the displacement of the NAO Platform.

\section{RESULTS}

The behavior of automatic speech processing and recognition by the NAO Robotics platform reflected $100 \%$ effectiveness associated with the validation of simple action commands such as "Walk", "Turn", "Stop" or "Greet"; this result was directly linked to the recognition of simple (single word) instructions; However, the recognition effectiveness was affected when using commands composed by several words such as "Raise right arm", "Lower left arm", "Walk forward 3 steps", "Turn right 45 degrees", among other predetermined composed instructions that were used with the Robotics platform. In this last case, there was a $23 \%$ margin of error with respect to the tests carried out.

With regard to processing linked to speech recognition, the average response time of the NAO robotic platform was 25 milliseconds; no measurements were made on the mechanical displacements of the platform, as they always worked correctly and are directly linked to the configuration parameters of the locomotion methods provided by the manufacturer's API.

\section{CONCLUSIONS}

An effective mechanism for the control and manipulation of the NAO robotic platform was presented, based on automatic VOICE recognition through the use of the Sphinx CMU library, capable of allowing a fluid interaction through verbal language between the robotic platform and its respective user. Predetermined action commands were validated that allowed control over locomotion and the execution of simple actions in processes of displacement and reproduction of messages that can be used in diverse processes of human-robot interaction; be it socialization, entertainment, education, among many others. With respect to the locomotion and movement control process of the platform, the functionality of the solution 
presented was demonstrated, so that it can be used as a reference and starting point in the development of new works related to the use of the NAO humanoid platform.

\section{REFERENCES}

1. Amit, T. (2019). Introduction to Hidden Markov Models. Retrieved from https://towardsdatascience.com/introduction-tohidden-markov-models-cd2c93e6b781

2. Andrist, S., Tan, X. Z., Gleicher, M., \& Mutlu, B. (2014). Conversational gaze aversion for humanlike robots. In 20149 th ACM/IEEE International Conference on Human-Robot Interaction (HRI), 25-32.

3. Asemi, A., Salim, S. S. B., Shahamiri, S. R., Asemi, A., \& Houshangi, N. (2019). Adaptive neuro-fuzzy inference system for evaluating dysarthric automatic speech recognition (ASR) systems: a case study on MVML-based ASR. Soft Computing, 23(10), 3529-3544.

4. Burget, F., Fiederer, L. D. J., Kuhner, D., Völker, M., Aldinger, J., Schirrmeister, R. T., ... \& Burgard, W. (2017, September). Acting thoughts: Towards a mobile robotic service assistant for users with limited communication skills. In 2017 European Conference on Mobile Robots (ECMR), 1-6.

5. Castro, D. (2011). Prototipo de un reconocedor de voz para el idioma español. Universidad Central de Venezuela, Caracas, Venezuela.

6. Huang, C. M., \& Mutlu, B. (2014). Learning-based modeling of multimodal behaviors for humanlike robots. In 20149 th ACM/IEEE International Conference on Human-Robot Interaction (HRI), 57-64.

7. Kennedy, J., Lemaignan, S., Montassier, C., Lavalade, P., Irfan, B., Papadopoulos, F., ... \& Belpaeme, T. (2017, March). Child speech recognition in human-robot interaction: evaluations and recommendations. In Proceedings of the 2017 ACM/IEEE International Conference on Human-Robot Interaction, 82-90.

8. Keselj, V. (2009). Speech and Language Processing Daniel Jurafsky and James H. Martin (Stanford University and University of Colorado at Boulder) Pearson Prentice Hall, hardbound, ISBN 978-0-13-187321-6.

9. Kitakoshi, D., Okano, T., \& Suzuki, M. (2017). An empirical study on evaluating basic characteristics and adaptability to users of a preventive care system with learning communication robots. Soft computing, 21(2), 331-351.

10. Kozhirbayev, Z., Erol, B. A., Sharipbay, A., \& Jamshidi, M. (2018). Speaker recognition for robotic control via an IoT device. In 2018 World Automation Congress (WAC), 1-5.

11. Maldonado, D., Villalba, R., \& Pinto, D. (2016). Sistema de reconocimiento automático del habla en guaraní. In Simposio Argentino de Inteligencia Artificial (ASAI 2016)-JAIIO 45.

12. Montiel, H., Martínez, F. \& Jacinto, E. (2017). Visual patterns recognition in robotic platforms through the use of neural networks and image processing. International Journal of Applied Engineering Research, 12(18), 7770-7774.

13. Nasereddin, H. H., \& Omari, A. A. R. (2017, July). Classification techniques for automatic speech recognition (ASR) algorithms used with real time speech translation. In 2017 Computing Conference, 200-207.

14. Rosen, E., Whitney, D., Phillips, E., Chien, G., Tompkin, J., Konidaris, G., \& Tellex, S. (2019). Communicating and controlling robot arm motion intent through mixed-reality head-mounted displays. The International Journal of Robotics Research, 38(12-13), 1513-1526.

15. Sauppé, A., \& Mutlu, B. (2014a). Design patterns for exploring and prototyping human-robot interactions. In Proceedings of the SIGCHI Conference on Human Factors in Computing Systems, 1439-1448. 
16. Sauppé, A., \& Mutlu, B. (2014b). How social cues shape task coordination and communication. In Proceedings of the 17th ACM conference on Computer supported cooperative work \& social computing, 97-108.

17. Sauppé, A., \& Mutlu, B. (2014c). Robot deictics: How gesture and context shape referential communication. In 20149 th ACM/IEEE International Conference on Human-Robot Interaction (HRI), 342-349.

18. Softbankrobotics (2020a). NAO Developer Guide. Retrieved from Https://developer.softbankrobotics.com/nao6/naodocumentation/nao-developer-guide

19. Softbankrobotics (2020b). NAO User Guide. Retrieved from https://developer.softbankrobotics.com/nao6/naodocumentation/nao-user-guide

20. UPase, S. U. (2016). Speech recognition based robotic system of wheelchair for disable people. In 2016 International Conference on Communication and Electronics Systems (ICCES), 1-5. 